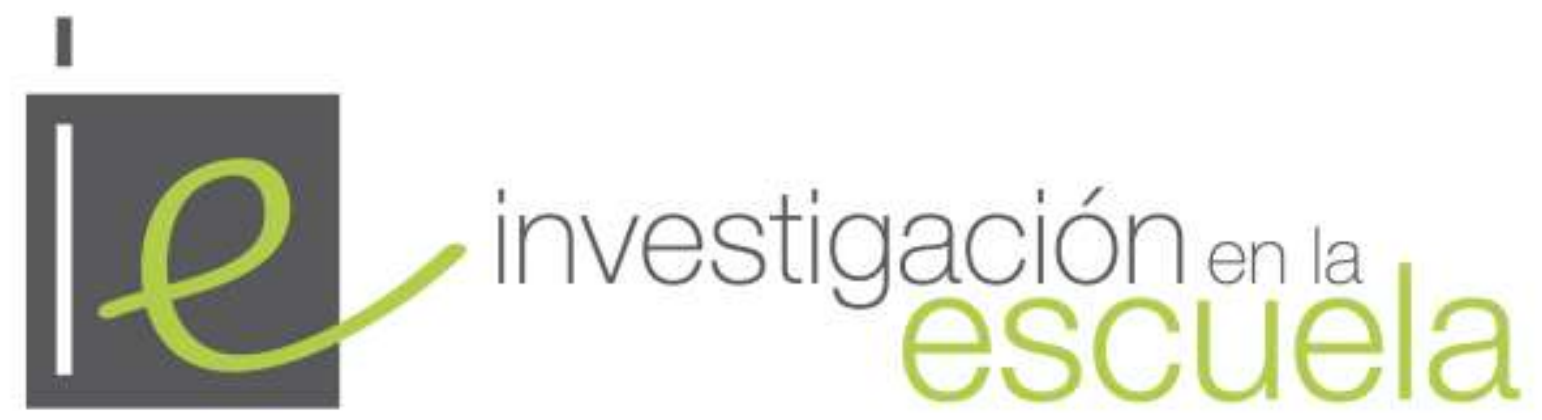

Revista internacional de investigación e innovación educativa

\title{
La enseñanza de la Historia en el bachillerato y su devenir innovador
}

\author{
Olga Duarte Piña \\ Universidad de Sevilla \\ España
}

Citación: Duarte-Piña, O. (2018). La enseñanza de la Historia en el bachillerato y su devenir innovador. Investigación en la Escuela, 96, 67-79.

Resumen: Los planteamientos metodológicos y los proyectos curriculares vinculados a la enseñanza de la Historia dependerán de los contextos políticos y educativos, de los profesores, de las corrientes de pensamiento que han influido en la didáctica, de la finalidad educativa y el valor social atribuido. Por tanto, reflexionar sobre la enseñanza de la Historia y su innovación didáctica, haciendo confluir todos estos planos, interesaría para mejorar la práctica, la de hoy y la que vendrá, y dejar constancia de su devenir innovador. Esta ha sido la principal finalidad del trabajo que se presenta, en el que se ha estudiado a profesores y grupos de innovación que sostuvieron propuestas de cambio frente a una enseñanza dela Historia basada en la trasmisión y memorización de contenidos principalmente políticos, organizados cronológicamente y centrados en el ámbito del occidente europeo.

Palabras clave: "Enseñanza de la Historia"; "innovación pedagógica"; "desarrollo de la educación”; "enseñanza secundaria".

The teaching of History in the baccalaureate and its innovative future

Abstract: Methodological approaches and curricular projects linked to the teaching of history will depend on the political and educational contexts, the teachers, the currents of thought that have influenced didactics, the educational purpose and the social value attributed. Therefore, to reflect on the teaching of history and its didactic innovation, bringing all these levels together, would be of 
interest in order to improve the practice, the practice of today and the one to come, and to record its innovative future. This has been the main purpose of the work presented, in which teachers and innovation groups have been studied and supported proposals for change as opposed to a teaching of history based on the transmission and memorization of contents mainly political, organized chronologically and centered in the area of Western Europe.

Key words: "History instruction"; "pedagogical innovation"; "education development"; "secondary education".

\section{L'enseignement de l'Histoire au baccalauréat et son avenir novateur}

Resumè: Les approches méthodologiques et les projets curriculaires liés à l'enseignement de l'histoire dépendront des contextes politiques et éducatifs, des enseignants, des courants de pensée qui ont influencé la didactique, du but éducatif et de la valeur sociale attribuée. Il serait donc intéressant de réfléchir à l'enseignement de l'histoire et à son innovation didactique, en rassemblant tous ces niveaux, afin d'améliorer la pratique, celle d'aujourd'hui et celle de demain, et d'enregistrer son avenir novateur. Tel a été le principal objectif du travail présenté, dans lequel les enseignants et les groupes d'innovation ont été étudiés et ont soutenu des propositions de changement par opposition à un enseignement de l'histoire fondé sur la transmission et la mémorisation de contenus principalement politiques, organisés chronologiquement et centrés dans le domaine de l'Europe occidentale.

Mots-clés: "Enseignement de l'histoire "; " innovation pédagogique "; " développement de l'éducation "; " enseignement secondaire".

\section{Introducción}

Dejó escrito Ortega y Gasset (2014, p. 215) "El hombre no tiene naturaleza, sino que tiene... historia", así que, por esta importancia consustancial al ser humano, pero también por su discurrir desde la ciencia a la enseñanza, la Historia debe ser investigada.

En este trabajo abordamos distintos planos que convergen para explicar la innovación en una disciplina que aparece como asignatura a mediados del siglo XIX, concretamente entre 1836 (Plan del Duque de Rivas y Arreglo Provisional) y 1838 (Ley de Instrucción Primaria). En estos momentos se concibe y aprueba la inclusión de la Historia en la educación primaria de grado superior y en la segunda enseñanza, especialmente el conocimiento de los hechos políticos siguiendo su cronología y particularmente la Historia de España (Cuesta Fernández, 1998).

Remontando el tiempo en que la disciplina es asignatura, el nudo gordiano de su enseñanza, caracterizada por una Historia lineal, fragmentada en edades, centrada principalmente en hechos y personajes políticos y en un aprendizaje memorístico, desconectado de la realidad del alumnado, se ha intentado desatar varias veces, pero nunca se ha conseguido deshacer definitivamente.

La innovación ha intervenido para desatar ese nudo gordiano. Como acto de subversión de la tradición, la innovación es consustancial a la educación, pero no trasciende ni transforma sustantivamente el plano de referencia o plano de organización escolar. Además, responde al principio de multiplicidad y, por ello, genera formas diferentes de enseñar y aprender dependiendo de los profesores y sus metodologías, de los contextos culturales, de las instituciones y políticas educativas, también, de las publicaciones que se difunden. En este sentido, la actividad innovadora es un proceso de cualificación continua que no conforma estructura ni jerarquía, lo que pretende cualquier reforma educativa. Esta variabilidad de las innovaciones supone multiplicidades de transformación que cambian de naturaleza al conectarse con otras y que, al ser líneas de fuga, pueden interrumpirse pero 
siempre recomienzan ${ }^{1}$. Cada innovación echa sus propias raíces, constituyendo rizomas, arraiga en determinadas circunstancias y colectivos afines al cambio, a un cambio educativo, pero también social y cultural, que crea relaciones con distintas intensidades y velocidades. Son desviaciones, lo opuesto a la versión única que constituyen las reformas y han supuesto procesos de transformación para la enseñanza de la Historia. No obstante, la innovación ha sido constante, aunque discontinua y lo esencial de esta discontinuidad, en la que ha adoptado diferentes representaciones, es que surge tras la experiencia docente porque es inmanente a la enseñanza. Este deseo de innovar genera una cultura innovadora que cambia con las generaciones y las epistemes ${ }^{2}$, también cambia porque el sistema educativo en el que se desarrolla no es el mismo, si bien el deseo de innovar la enseñanza de la Historia ha mantenido una misma orientación: que se estudie en su carácter holístico y sea útil para la formación social y ciudadana del alumnado, que desarrolle su pensamiento crítico, consiguiendo llegar al pasado, para comprender el presente y avanzar un proyecto de futuro (Gruzinski, 2018).

La investigación que sostiene este trabajo ${ }^{3}$ tiene como finalidad realizar un recorrido por otras formas de entender y plantear la enseñanza de la Historia. Este recorrido se inicia a fines del siglo XIX con Rafael Altamira, continúa con los catedráticos de instituto Rafael Ballester y Castell y Antonio Jaén Morente, y los profesores de Escuela Normal Teófilo Sanjuán Bartolomé, Gloria Giner de los Ríos García y Daniel González-Linacero ${ }^{4}$. Con sus propuestas innovadoras se evidencia el deseo de resolver ese nudo gordiano evidenciando, en sus publicaciones, cómo conseguir que la enseñanza de la Historia deje de ser memorística, erudita, irrelevante para el aprendizaje del alumnado y desconectada del presente. Igualmente sucederá con diferentes grupos de innovación en el último tercio del siglo XX. De estos grupos ${ }^{5}$ están los que constituyeron paradigma innovador a mediados de los años 70, Germanía 75 e Historia 13-16 y dos ejemplos destacados como el proyecto editorial de Adara y el de Grupo de Humanística para la Formación Profesional; los proyectos curriculares de los grupos Cronos, Aula Sete, Ínsula Barataria y Bitácora, que ganaron una convocatoria en 1990 del Ministerio de Educación para la elaboración de materiales de enseñanza, y más grupos que se constituyeron, en estos años, aunque, como testigos activos de una forma nueva de entender la enseñanza de la Historia, hoy en día, quedan Grupo de Investigación y Renovación Escolar (IRES) y Gea-Clío.

Para desentrañar el devenir-innovador ${ }^{6}$ de la enseñanza de la Historia, acudimos a tres líneas que podrían explicarlo. La línea de los factores que propician la innovación: los contextos políticoeducativo y cultural, las agencias productoras de discurso y los agentes (profesores e instituciones

\footnotetext{
${ }^{1}$ Los conceptos en cursiva pertenecen a los planteamientos teóricos sobre el concepto de rizoma de Deleuze y Guattari (2010) y los utilizamos para explicar el concepto de innovación en la enseñanza.

${ }^{2}$ Episteme según Foucault (2012) muestra los saberes y relaciones de una época en su variabilidad y posibilidad, en sus conexiones u oposiciones.

${ }^{3}$ Duarte Piña, Olga (2015). La enseñanza de la Historia en la Educación Secundaria: innovación, cambio y continuidad. Tesis doctoral inédita dirigida por Dr. D. F. Javier Merchán Iglesias. Universidad de Sevilla.

${ }^{4}$ Los autores elegidos, profesores en distintas instituciones como el Centro de Estudios Históricos, la Escuela de Estudios Superiores del Magisterio e Institutos de Bachillerato, lo han sido porque constituyen un primer referente de innovación y por el alcance de la difusión de sus propuestas de cambio educativo. En el anexo 4 de Mainer Baqué (2009, pp. 759-761 y pp. 787-791) están catalogadas dichas propuestas como Textos declarativos de la Didáctica de las Ciencias Sociales. Primer orden y Textos-manuales para la escuela.

${ }^{5}$ De los grupos de innovación referidos fueron entrevistados o respondieron a un cuestionario, miembros destacados de los mismos, en el marco de la investigación de tesis doctoral del que deriva este artículo.

${ }^{6}$ Devenir no es llegar a ser, ni evolucionar, no es dependencia sino creación, es multiplicidad, divergencia; devenir "es un verbo que tiene toda su consistencia; no se puede reducir, y no nos conduce a "parecer", ni "ser", ni "equivaler", ni "producir"” (Deleuze y Guattari, 2010, p. 245). Aplicado este verbo a la innovación nos permite explicar su expansión, heterogeneidad, regresión, transformación, comunicación.
} 
que dieron cabida y difusión a la actividad innovadora) y las corrientes de pensamiento histórico; la línea de las características que presenta la innovación (modelo y proyecto de enseñanza, valor y utilidad del conocimiento histórico); y la línea de las repercusiones, evidenciada en cambios y continuidades. Para abordar estas líneas, a continuación, realizamos un sintético análisis.

\section{Los factores que propician la innovación en la enseñanza de la Historia}

\section{Los contextos políticos y educativos}

Los contextos de cambio político suponen impulsos para la innovación. Así, en el tránsito finisecular y el primer tercio del siglo XX, se produjo una confluencia entre la cultura institucional y pedagógica, en términos de Escolano (2002), que favoreció la innovación. Un precedente fue la alianza entre pedagogía y krausismo durante el Sexenio revolucionario (Ruiz Torres, 2001) que influiría en la creación de la Institución Libre de Enseñanza fundada en 1876.

En los inicios del siglo XX, se aprobaron planes de estudio para el bachillerato que contribuyeron a mejorarlo: el Plan de estudios de bachillerato de 1903, los primeros cuestionarios unificados para todo el territorio nacional en 1927 y el Plan de bachillerato de 1934. En el contexto político, tanto el regeneracionismo como el socialismo republicano tuvieron intenciones claras de cambiar una realidad nacional confiándose el cambio al desarrollo del sistema educativo. Hubo, en estas primeras décadas, una coincidencia entre la cultura institucional y la cultura pedagógica, y en la II República concordaron ampliamente los intereses de las tres culturas: institucional, pedagógica y práctica (Escolano, 2002), encarnadas en políticos, intelectuales, pedagogos y profesores. Ejemplos de lo dicho, el libro de Rafael Ballester Clío en las aulas (1913) que sigue el Plan de estudios de 1903; Rafael Altamira publica en 1927 Epitome de Historia de España (Libro para los profesores y maestros) y Antonio Jaén Morente recoge la reforma de los contenidos del Plan de 1934 en su manual Lecturas históricas (Iniciación) editado en 1935.

Durante la edad oscura (1945-1958) ${ }^{7}$ hubo reformas y planes de estudio para extraer del sistema cualquier traza de lo anterior. A mediados de los 50, la evolución socioeconómica española ${ }^{8}$ permitiría progresivamente cambios en los distintos niveles educativos. Como exponentes y cauces de apertura, el Centro de Orientación Didáctica para la Enseñanza Media y el Centro de Documentación y Orientación Didáctica de la Enseñanza Primaria (CEDODEP) y diversas revistas, entre ellas, Enseñanza Media y Vida Escolar (Mainer Baqué, 2009).

Hacia fines de los años sesenta se constata el tránsito del modo tradicional-elitista al tecnocrático de masas (Cuesta Fernández, 1998) y la "llegada masiva al profesorado oficial de una generación de posguerra curtida en los movimientos antifranquistas" (Zaragoza, 1997, p. 170).

La Ley General de Educación de 1970 reconoció la necesidad de adecuar el sistema educativo a una sociedad que se había ido transformando. El impulso principal de la innovación, aun en un contexto poco favorable, fue utilizado como espoleta para el cambio de paradigma educativo y cultural. La innovación se sitúa en la vanguardia, porque el acto de innovar resulta del diagnóstico de la realidad para renovarla, y, para la enseñanza de la Historia, surgen los grupos Germanía 75,

\footnotetext{
7 “Con la escasa, pero relevante, excepción de los Cuestionarios, el tiempo transcurrido entre la aprobación de la Ley de 1945 y la creación del CEDODEP representó una especie de edad oscura, desde el punto de vista curricular, en que nada significativo ocurría en el panorama español” (Beltrán Llavador, 1991, p. 127).

8 "Tras el prolongado periodo autárquico de la posguerra, será a mediados de los años cincuenta cuando aparezcan los primeros rasgos que apuntaban hacia una liberalización y modernización del sistema económico. Hechos como la composición del quinto gobierno franquista (1957), con la entrada de varios ministros pertenecientes al Opus Dei, la reforma de la Administración realizada en 1957 y el Plan de Estabilización llevado a cabo en 1959, han sido interpretados como expresiones de la adopción de un modelo de desarrollo que diese respuesta a determinados problemas que tenía planteados la sociedad española del momento” (Luis y Rozada, 1997, p. 243).
} 
Historia 13-16, Grupo de Humanística, la primera propuesta de Grupo Cronos y el proyecto de la editorial Adara.

En los inicios de la democracia se descubren los discursos innovadores del primer tercio del siglo XX y empieza a difundirse el conocimiento innovador en investigaciones, estudios y experiencias. El cambio educativo iba a la par del cambio político y social, al igual que en el periodo de la Restauración y el de la II República, pero implantada, en 1990, la Ley Orgánica General del Sistema Educativo (LOGSE), el impulso dado por los proyectos curriculares de los grupos que concursaron en la convocatoria del Ministerios de Educación y Ciencia de 1990, Cronos, Ínsula Barataria, Aula Sete y Bitácora, más otros que habían surgido como Asklepios, IRES, Gea-Clío, Kairós, Pagadi, Espacio y Sociedad, empieza a declinar porque la innovación fue institucionalizándose en campos de poder ajenos al saber práctico de las aulas. Más adelante se comenta qué cauces para su formación y acción encuentran los grupos mencionados, dando continuidad a la actividad innovadora, algunos de ellos hasta la actualidad.

\section{Las agencias productoras de discurso}

Las agencias promoverán la formación docente, la innovación y la generación de nuevos discursos para la enseñanza de la Historia y las publicaciones difundirán las nuevas propuestas. A principios de la centuria están el Centro de Estudios Históricos $(\mathrm{CEH})$, donde Rafael Altamira dirigió la "Sección de Metodología de la Historia"; la Junta para la Ampliación de Estudios (JAE), con la concesión de pensiones para viajar y conocer cómo se desarrollaba la enseñanza de la Historia en otros países de nuestro entorno; la Escuela de Estudios Superiores de Magisterio (EESM) y la Cátedra de Pedagogía Superior en la Universidad Central de Madrid. Son las primeras agencias productoras de discurso (Mainer Baqué, 2009) y en estas agencias se formarán o se desarrollarán profesionalmente profesores afanados en proponer metodologías innovadoras de enseñanza de la Historia. Antonio Jaén fue alumno de Rafael Altamira en el CEH y becado por la JAE, al igual que Rafael Ballester y Gloria Giner; ésta junto a Teófilo Sanjuán y Daniel González-Linacero serán alumnos de la ESSM y también profesores de Escuela Normal.

En los años sesenta pueden caracterizarse otras agencias productoras de discurso. Destaca la Universidad Literaria de Valencia donde, en la Facultad de Filosofía y Letras, se formaron los miembros del grupo Germanía 75, pero también las de Barcelona, Salamanca, Sevilla o Madrid. En los setenta, los Colegios de Doctores y Licenciados, los Institutos de Ciencias de la Educación, los Movimientos de Renovación Pedagógica, las Escuelas de Verano y, en los ochenta, los Centros de Profesorado. A mediados de los años noventa, la constitución de la Federación Icaria (Fedicaria) va a suponer un nuevo tipo de agencia productora de discurso, no desde el ámbito de las instituciones públicas, aunque su labor intelectual y profesional se hará con un fin público.

Las agencias contextualizarán y promoverán la innovación, sirviendo de catalizador y amplificador. Si en el albor de la nueva enseñanza de la Historia, y entre las décadas de los 70 y hasta fines de los años 90, las agencias productoras de discurso fueron centros de formación del profesorado, hoy las agencias son centros de emisión de méritos y la formación no se articula, adecuadamente, ni a través de éstas ni en la formación inicial de los licenciados en Historia. Pudo haberse hecho durante el periodo de Reforma iniciado en los años ochenta que culminó con la aprobación de la Ley Orgánica General del Sistema Educativo (LOGSE), demandándose tanto por algunos asesores de los Centros de Profesorado como por los grupos innovadores, pero se actuó desde la macroestructura política, la reforma desde arriba (Coll y Porlán, 1998; Rozada, 2003) anulando la vocación hacia la innovación desde la propia experiencia. A partir de entonces, "desprestigiados los movimientos de reforma y anulados los grupos de experimentación, el sistema quedó inválido de ideología y carente de racionalidad práctica" (Gimeno Sacristán, 2007, p. 39). No obstante, los grupos de innovación, constituidos en este periodo, atendieron sus necesidades formativas en lo que 
llamaron Seminarios sobre Desarrollo Curricular en el Área de CC.SS., Geografía e Historia. Educación Secundaria a través de un programa de investigación educativa, de elaboración de materiales y de experimentación de las propuestas. Estos seminarios se celebraron entre 1990 y 1995, dándose continuidad a la idea, a partir de esta última fecha, en la Federación Icaria (Fedicaria) que, en 2013, iniciaría una nueva singladura más centrada en una perspectiva política de la educación.

\section{Las corrientes de pensamiento histórico}

"Historiografía y didáctica aparecen (...) como insoslayables caras de una misma moneda en cualquier proyecto renovador de ayer, de hoy y de mañana" (Mainer y Cuesta, 1997, p. 10). Pero las relaciones entre historiografía y didáctica han cambiado a medida que la didáctica específica de la Historia se ha desarrollado.

La historiografía académica positivista traducía a los programas de enseñanza un resumen de la Historia que se investigaba. En reacción a esta trasposición, Rafael Altamira miró a la Escuela Metódica francesa interesada en la ciencia y la enseñanza de la Historia.

Altamira en su visita a la Facultad de Letras de París y otros centros científicos, en el curso 1889-1890, conoce los planteamientos organizativos y pedagógicos y crea un círculo de intercambios y relaciones entre historiadores y profesores, ciencia y sociedad, historia y enseñanza, pasado y problemas del presente, estableciéndose las conexiones entre historiografía y didáctica desde la perspectiva de la función social del historiador-profesor (Maestro González, 2000). En los años 50 habrá una nueva influencia de la historiografía francesa a través de Vicens-Vives?. También, la historiografía académica de Annales trajo las renovaciones curriculares como la de 1970 (Valdeón, 1988) ${ }^{10}$ y la corriente materialista francesa repercutió en la selección de contenidos y propuestas metodológicas de los grupos Germanía 75, Cronos o Grupo de Humanística.

Cuando Grupo Cronos realiza su primera programación renovada reconoce "la penuria teórica de la que partimos a la hora de fundamentar, desde las ciencias de la educación, una alternativa de didáctica de la Historia coherente" (Baigorri, Castán, Cuesta, Cuadrado, Gómez y López, 1984, p. 10). Sin embargo, cuando en los 90 presentan su Proyecto Cronos. Ciencias Sociales, Historia y Geografía, ya no está como referente la investigación historiográfica sino la investigación educativa que había avanzado en ese tiempo. Además, desde los años ochenta, se consolidó en las universidades el campo de la Didáctica de las Ciencias Sociales que fue "desplazando progresivamente a una indagación didáctica más vinculada a las iniciativas espontáneas y desinstitucionalizadas de los grupos y movimientos de renovación pedagógica" (Cuesta Fernández, 2001, p. 106).

Si la historiografía tuvo en el pasado un papel revulsivo de cara a la innovación, ahora no lo tiene.

Hoy, después de unos años de transformación y de progreso indudablemente de las prácticas y las doctrinas del historiar, estamos en una situación en la que no se producen hallazgos de suficiente generalidad como para que representen vías plausibles para ulterior avance. Lo que el panorama muestra es una cierta detención de las innovaciones, un cierto escolasticismo temático y formalista, volcado a veces hacia la historia de trivialidades — la historia light_, un neonarrativismo, aun cuando con cierta inclinación etnológica, que tiene

\footnotetext{
${ }^{9}$ Miquel Tarradell, Joan Reglá y Emili Giralt, discípulos de Vicens Vives, serán profesores en la Facultad de Filosofía y Letras de Valencia, a mediados de los años sesenta, de quienes en los setenta fundarán Germanía 75 e Historia 13-16.

${ }^{10}$ El historiador lo describe con estas palabras. “(...) los programas de las asignaturas de Historia del Bachillerato, implantados en la década de los setenta, sancionaban el entierro de la vieja historia. En su lugar se imponía una "historia de las civilizaciones" que debía mucho a Braudel y a la escuela francesa de los “Annales”” (Valdeón, 1988, p. 9).
} 
más de revival, efectivamente, que de innovación, el interminable epigonismo de la historiografía francesa de los Annales, cuando no esa especie de huida hacia adelante que parecen significar algunas posiciones recientes más diletantes que efectivas. (Aróstegui, 1995, p. 34).

Esta etapa comenzó vinculando el cambio de la enseñanza de la Historia a las cuestiones metodológicas como exponente de la innovación, vinculación que llega hasta principios de los noventa, cuando aparecen las propuestas desarrolladas en los proyectos curriculares de los grupos de innovación. En los proyectos se entendía la didáctica como un sistema en el que integrar los contenidos, la metodología, la evaluación y las finalidades de la enseñanza. Los proyectos, además, venían a establecer los puentes necesarios entre la teoría educativa y la práctica para el desarrollo del currículo y de la formación docente. Sin embargo, la fundamentación definitiva de la Reforma no dio validez a esta proyección de los proyectos curriculares y optó, de nuevo, por una Historia fragmentada, organizada por periodos que se corresponden a cursos (Prehistoria a Medievo, Modernidad y Contemporaneidad) y reducida a sociedades históricas que suponían "un sucedáno de historia cultural [identificada] con una galería de personajes ilustres” (Gruzinski, 2018, p. 61).

Hacia el final de la centuria, los grupos de innovación defendieron una nueva

fundamentación de la enseñanza de la Historia desde una base originaria que definía la disciplina en su dimensión escolar, en su dependencia curricular y con una eficaz utilidad social y formativa. Esta nueva fundamentación, consensuada en el seno de Fedicaria, enuncia cinco principios orientadores (problematizar el presente, pensar históricamente, educar el deseo, aprender dialogando e impugnar los códigos pedagógicos y profesionales) y tres estrategias centradas en la producción y usos de los materiales escolares, la desactivación de los espacios y tiempos impuestos por la institución escolar y la problematización de la raíces identitarias del profesorado y alumnado en tanto que sujetos constituidos y constituyentes de la realidad escolar (Cuesta Fernández, 2007, p. 46 y p. 62).

\section{Los agentes}

La generación de 1868 caracterizada como la del hombre nuevo (Millán Chivite, 1979, p. 24) va a tener en la educación un eje de acción. Esta generación será impulsora de reformas significativas y será referente para profesores de momentos posteriores; ya en los primeros años del siglo XX están Rafael Altamira y Rafael Ballester como padres fundadores (Mainer Baqué, 2009) y los continuadores-discípulos de esta generación, mencionados anteriormente.

Aprovechando una coincidencia numérica, quizá casual, citamos una explicación que nos permite establecer un paralelismo generacional entre aquellos albores de la innovación y la que retorna en grupos de profesores a mediados de los años setenta del siglo XX, es decir, justo un siglo después.

(...) que una buena porción de los profesores de Historia de BUP no haya puesto reparos a la renovación de los contenidos históricos que se opera en los textos visibles de la nueva educación histórica iniciada en los planes de mediados de los setenta, y que, asimismo, en el conjunto profesional subyazca un componente ideológico avanzado. De ahí que la impregnación ideológica y las retóricas progresistas no sean, ni mucho menos, ajenas a una parte considerable de los profesores de Historia de la "generación del 68" (Cuesta Fernández, 1998, p. 174).

Todo este trabajo dedicado a la innovación está encarnado en los profesores que van a estar en las agencias productoras de discurso, implicados en los procesos de cambio y transformación, pensadores y hacedores de otra enseñanza de la Historia. Siempre profesores, historiadores o pedagogos que, a mediados de los ochenta, fueron progresivamente desplazados por psicólogos, 
técnicos y asesores que se apropiaron y atomizaron la innovación educativa en campos de poder (Bourdieu, 2002), diferenciándose, por una parte, una innovación institucionalizada alejada de la práctica docente y centrada en los medios y, por otra, una didáctica crítica, sostenida por los grupos de innovación, vinculada a la "función emancipadora y finalista del conocimiento histórico" (Cuesta Fernández, 1998, p. 217).

Se ha escrito sobre cuán importante es contar con el profesorado a la hora de las reformas educativas y, en todo el periodo estudiado, salvo en el breve lapso del primer bienio republicano (1931-1933), no han confluido las tres culturas de la escuela (institucional, pedagógica y práctica) en los momentos de cambio. En los años ochenta, desde la macroestructura educativa, se aplicó una reforma en un proceso descendente de arriba-abajo (Coll y Porlán, 1998), pensándose que se implicaría al profesorado. Pero, si durante el primer bienio republicano con un gobierno socialista hubo acercamiento entre las tres culturas escolares, con el primer gobierno socialista (1982), tras la dictadura, éstas iniciaron un distanciamiento que aún hoy permanece.

Según Jurjo Torres (2007) las reformas contribuyeron a la desprofesionalización del profesorado". Incluso la "invención institucional del campo de la didáctica especial de las Ciencias Sociales en el marco universitario" (Mainer Baqué, 2009, p. 11) ha venido a limitar la propia profesionalización del docente en Secundaria, al convertirse en un ámbito específico de producción de saber-poder con sus propios espacios de socialización profesional y divulgación. Aróstegui (1995, p. 36) viene a afirmar que "los historiadores salidos de nuestras universidades carecen, por lo común, de teoría y de método. La formación recibida es puramente memorística y más que mediocre", cuestión que ha ido agrandándose porque la Universidad de hoy en día, donde se forman los historiadores y de donde salen los futuros profesores, está caracterizada por "una vida intelectual reducida a las funciones curriculares, cada vez más tecnificadas y burocratizadas (...) orientada hacia las llamadas ciencias rentables” (Prats, 2016, p. 151), que apartan el valor de las Humanidades. En definitiva, sin una formación inicial y permanente, vinculadas a la finalidad educativa, al valor social de la disciplina y la mejora de su enseñanza, con escasos espacios para comunicar el desarrollo profesional docente, la potencialidad de la innovación no deviene verbo, sino que se sustantiva, favoreciendo la continuidad de modelos y métodos tradicionales.

\section{Las características de una nueva forma de enseñanza la Historia}

En una cualificación discontinua de la innovación, la enseñanza de la Historia, aunque en su origen se concibiera maestra para la vida y clarificadora del presente, llegó a ser un instrumento para el cambio social y cultural, un método para el desarrollo del intelecto y una materia para la alfabetización cultural y crítica. Todos estas formas se han ido incorporando ${ }^{12}$; mas, la narración

\footnotetext{
11 “(...) puede decirse que el PP contribuyó a desprestigiar mucho al profesorado, en especial al de la red pública. (...) Si ya apenas tenían autonomía, se la recortaron más. Recuerda que el PSOE había creado los CEPs, que al principio tenían bastante autonomía para programar y organizar actividades, aunque luego, como ya dije, cometió el error de poner al frente a alguien que precisaba el carnet del PSOE o de la UGT, y llegó un momento en que impuso un mayor control sobre ellos, recortándoles autonomía. Cuando entró la derecha esa tendencia intervencionista se acentuó aún más" (Torres, 2007, p. 139).

12 “(...) el argumento de la Historia como maestra de la vida de la que se pueden tomar "lecciones" sigue presente cuando se destaca el valor cívico de la disciplina, sólo que ahora, en lugar de cargar las tintas sobre episodios y personajes ejemplares, se prefiere la denostación de hechos históricos contrarios a los valores dominantes, centrándose más en la capacidad de la Historia para hacer ver lo que debe evitarse que para seguir modelos de conducta (Merchán Iglesias, 2005, p. 30). La Historia para comprender el presente se ha convertido en la piedra angular de la arquitectura del discurso sobre la asignatura" (Cuesta Fernández, 1998, p. 32), dando por hecho que sólo por estudiar, memorizar o comprender el pasado, los alumnos van a entender el presente.
} 
cronológica de hechos políticos e institucionales, la identificación con la nación o naciones, la memoria como potencia formativa, el libro de texto como representación ilustrada de los contenidos programados constituyen, desde antaño a hoy en día, el código disciplinar de la segunda enseñanza (Cuesta Fernández, 1998), que continúa en vigor.

Altamira abogó por un periodo de cultura general que evitaría el estudio de los mismos temas en las etapas Primaria y en Secundaria. En los años 70, los primeros grupos de innovación propusieron una enseñanza de la Historia que contribuyera al cambio social y cultural y los proyectos curriculares, de los grupos de innovación de los años 90, fueron alternativas muy bien planteadas frente a una tradición que venía reiterándose, herramientas para la formación docente y para la alfabetización cultural y crítica del alumnado. Sin embargo, en los planes actuales permanece la organización cronológica de una Historia principalmente política, memorística y culturalista.

La reducción de contenidos, iniciada en los Cuestionarios de 1954, que llega a la LOGSE (1990), facilitó el arbitrio editorial, encargándose las empresas editoras de la programación de los contenidos a enseñar, generando esto un crecimiento desmesurado y progresivo del mercado de libros de texto y provocando lo que podría decirse, y vuelvo a transcribir, en palabras de Altamira casi cien años después:

Así, en lugar de decrecer el valor instrumental del libro de texto en la primera y segunda enseñanza, ha crecido hasta convertirse en el eje de esas enseñanzas, con grave peligro de suprimir al maestro mismo, único vivo de la didáctica, y único también en quien puede fundarse, si se le dirige y forma como es debido, una verdadera eficacia de la educación escolar (Altamira, 1927, p. 8).

Las innovaciones en sus modelos, finalidades y valores no avanzaron más allá de los círculos de profesores en los que se generaron, no consiguieron cambiar la selección de los contenidos a enseñar, ni la metodología transmisiva ni las actividades para el aprendizaje. Hoy la Historia enseñada poco se diferencia de la que en diferentes contextos y por distintos agentes se ha intentado conculcar.

\section{Cambios y continuidades}

A lo largo de este trabajo, a pesar de su carácter sintético, han ido evidenciándose varias cuestiones: la labor de los docentes en la innovación para la enseñanza de la Historia, su implicación en contextos favorables y en momentos poco propicios; la investigación de los cauces por los que la ciencia ha de transformarse en conocimiento escolar, los obstáculos y las dificultades para la difusión de las propuestas, el surgimiento y el debilitamiento de la actividad innovadora que es devenir, una acción con líneas de actuación, velocidades a distintos ritmos y dimensiones según el contexto y los agentes. $\mathrm{Y}$, aunque estas líneas pueden romperse e interrumpirse, siempre recomienzan según esta o aquella de sus líneas o según otras, porque la innovación es inmanente, constante, aunque discontinua, y surge tras la experiencia de la enseñanza y el cuestionamiento de la misma para mejorarla.

No obstante, como afirma Merchán Iglesias (2005, p. 25), la Historia escolar es "el principal obstáculo en la formación histórica de los jóvenes". Y para que confluyan innovación y formación en la enseñanza de la Historia no ha de prevalecer la investigación histórica sobre la didáctica, creándose ámbitos paralelos: el del profesor y el historiador. Uno de los obstáculos de tal disyuntiva es que los docentes "prefieran ser considerados como expertos en un saber abstracto y académico -la Historia- que como expertos en un saber práctico -la enseñanza o la pedagogía-, como es el caso de 
los maestros, y que gozan de tan escaso prestigio en la sociedad" (op. cit., p. 38). Sólo en los profesores innovadores confluye la doble condición y la preocupación por el avance de la ciencia y de su enseñanza.

La ausencia de una formación didáctica inicial es una realidad y "forma parte también de la idiosincrasia de la profesión en este nivel de la educación secundaria" (Cuesta Fernández, 1998, p. 40). El profesor de secundaria se entiende con su propio saber y cree que puede transferirlo pero, como decía Sanjuán Bartolomé (1929, p. 11), "nadie mejor preparado que el investigador de los hechos para maestro de Historia". Es, por tanto, el sentido moral de la profesión, el entusiasmo y la vocación lo que han de conjuntarse para que el profesor sea un intelectual crítico.

Que lo profesores enseñan tal y como a ellos les enseñaron y que sus creencias dan forma a su actuación docente son afirmaciones constatadas desde Cuban (1984) a Porlán (2017), y si el aprendizaje de la Historia ha sido desde un modelo de enseñanza transmisivo, esto no explica por qué los protagonistas de este trabajo fueron capaces de generar cambios en la enseñanza de la Historia, aunque sí da respuesta a la continuidad y a la resistencia al cambio. Los cambios tuvieron que ver con la confluencia de múltiples factores que propiciaron la innovación y en este estudio se muestran. La innovación es cartografía, "si el mapa se opone al calco es precisamente porque está totalmente orientado hacia una experimentación que actúa sobre lo real” (Deleuze y Guattari, 2010, p. 18) y busca los cauces en donde otra enseñanza de la Historia es posible.

\section{Referencias}

Altamira, R. (1927). Epitome de Historia de España (Libro para los Profesores y Maestros). Madrid: Ediciones de la Lectura.

Aróstegui, J. (1995). La investigación histórica: teoría y método. Barcelona: Crítica.

Baigorri, J., Castán, G., Cuesta, R. F., Cuadrado, M., Gómez, F. y López, R.J.; Grupo CRONOS (1984). Historia de España (3º de B.U.P.). Libro del profesor. Salamanca: ICE/Ediciones de la Universidad de Salamanca.

Beltrán-Llavador, F. (1991). Política y reformas curriculares. Valencia: Servei de Publicacions de la Universitat de València.

Bourdieu, P. (2002). La distinción: Criterio y bases sociales del gusto. México: Taurus.

Coll, C. y Porlán, R. (1998). Alcance y perspectiva de una reforma educativa: la experiencia española. Investigación en la Escuela, 36, 5-29.

Cuban, L. (1984). How teachers taught. Constancy and change in american classrooms 1890-1980. New York: Longman (traducción F. J. Merchán Iglesias).

Cuesta-Fernández, R. (1998). Clío en las aulas. La enseñanza de la Historia en España entre reformas, ilusiones y rutinas. Madrid: Akal.

Cuesta-Fernández, R. (2001). La Didáctica de las Ciencias Sociales en España: un campo con fronteras. En J. Mainer (coord.). Discursos y prácticas para una didáctica de las CC. SS. Ideas y líneas de trabajo para transformar la enseñanza (pp. 103-115). Sevilla: Díada.

Cuesta-Fernández, R. (2007). Los deberes de la memoria en la educación. Barcelona: Octaedro.

Deleuze, G. y Guattari, F. (2010). Mil mesetas. Capitalismo y esquizofrenia. Valencia: Pre-textos.

Escolano-Benito, A. (2002). La educación en la España contemporánea. Políticas educativas, escolarización y culturas pedagógicas. Madrid: Biblioteca Nueva.

Foucault, M. (2012). Las palabras y las cosas. México: Siglo XXI.

Gimeno-Sacristán, J. (2007). Una visión desde dentro y desde fuera. En J. Varela. Las reformas educativas a debate (1982-2006), (pp. 19-44). Madrid: Morata.

Gruzinski, S. (2018). ¿Para qué sirve la Historia? Madrid: Alianza editorial. 
Luis, A. y Rozada J. M. (1997). La Renovación de la Enseñanza de la Geografía Española en la EGB (1970-1984): Nuevos proyectos y viejos problemas. En M. Carretero, J. I. Pozo y M. Asencio (comps). La enseñanza de las Ciencias Sociales (pp. 241-261). Madrid: Visor.

Maestro-González, P. (2000). Didáctica de la Historia, historiografía y enseñanza, 91-111. Íber. Didáctica de las Ciencias Sociales, Geografía e Historia 25.

Mainer-Baqué, J. (2009). La forja de un campo profesional. Pedagogía y didáctica de las Ciencias Sociales en España (1900-1970). Madrid: Consejo Superior de Investigaciones Científicas.

Mainer-Baqué, J. y Cuesta, R. (1997). Presentación. En R. Altamira. La enseñanza de la Historia (pp. 7 11). Madrid: Akal.

Merchán-Iglesias, J. (2005). Enseñanz̧a, examen y control. Profesores y alumnos en la clase de Historia.

Barcelona: Octaedro/Ediciones Universitarias de Barcelona.

Millán-Chivite, J. L. (1979). Revolucionarios, reformistas y reaccionarios. Sevilla: Universidad de Sevilla.

Ortega y Gasset, J. (2014). Historia como sistema. Madrid: Gredos.

Porlán, R. (coord.) (2017). Enseñanza universitaria. Cómo mejorarla. Madrid: Morata.

Prats-Cuevas, J. (2016). Combates por la Historia en la educación. Enseñanza de las Ciencias Sociales, $15,145-153$.

Ruiz-Torres, P. (2001). Política y ciencia de la Historia en la universidad de Valencia entre 1868 y 1939. Biblioteca Virtual IFC, Institución Fernando el Católico (pp. 55-95). Zaragoza: Institución

Fernando el Católico. Disponible en: http://ifc.dpz.es/recursos/publicaciones/22/93/04ruiz.pdf

Rozada, J. Ma (2003) (Coord.) Las reformas escolares de la democracia. Oviedo: Federación Icaria, Plataforma Asturiana de Educación Crítica y KRK Ediciones.

Sanjuán-Bartolomé, T. (1929). Cómo se enseña la Historia. Madrid: Publicaciones de la Revista de Pedagogía.

Torres, J. (2007). Las reformas contribuyeron a la desprofesionalización del profesorado. En J. Varela. Las reformas educativas a debate (1982-2006), (pp. 114-143). Madrid: Morata.

Valdeón-Baruque, J. (1988). En defensa de la Historia. Valladolid: Ámbito.

Zaragoza, G. (1997). La investigación y formación del pensamiento histórico del adolescente. En M. Carretero, J. I. Pozo y M. Asencio (comps.). La enseñanza de las Ciencias Sociales (pp. 165-177). Madrid: Visor.

\section{Información sobre los autores}

Autor: Olga Duarte Piña

Institución: Universidad de Sevilla.

Email: oduarte@us.es 


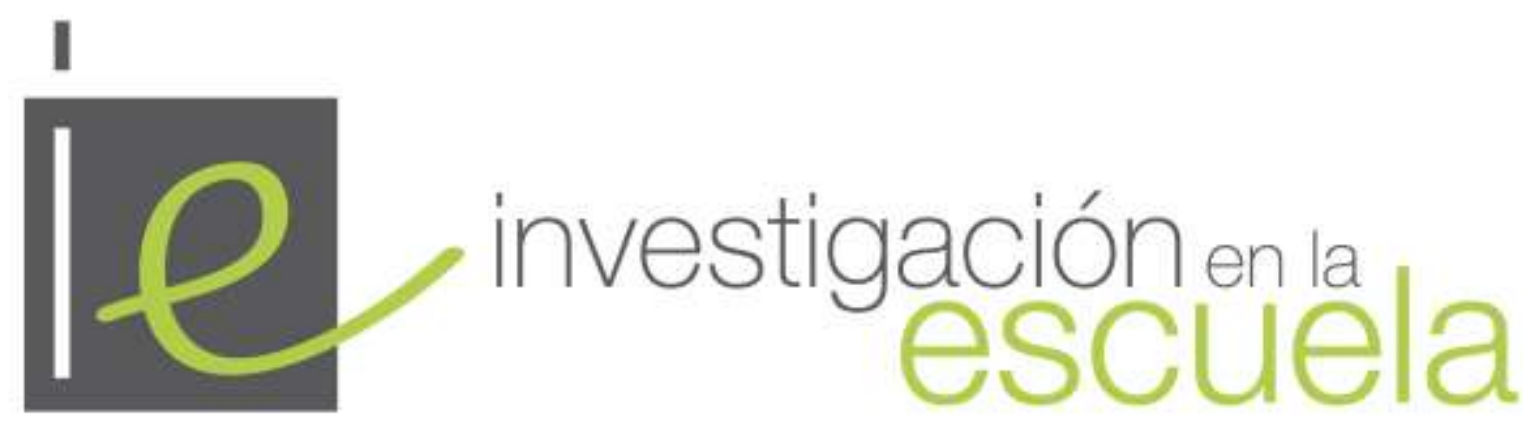

Revista académica evaluada por pares y de acceso abierto

Número 96

31 de diciembre de 2018

ISSN 2443-9991

(c)

SOMERIIGHISRESEEVEDLOS/as lectores/as pueden copiar, mostrar, y distribuir este artículo, siempre y cuando se de crédito y atribución al autor/es y a Investigación en la Escuela, se distribuya con propósitos no-comerciales, no se altere o transforme el trabajo original. Más detalles de la licencia de CreativeCommons se encuentran en http://creativecommons.org/licenses/by-nc-sa/3.0 Cualquier otro uso debe ser aprobado en conjunto por el autor/es, o Investigación en la Escuela.

Uit?

Revista Editada por la Universidad de Sevilla. https://editorial.us.es/es/revistainvestigacion-en-la-escuela

Contribuya con comentarios y sugerencias en la web de la revista. Por errores y sugerencias contacte a secretaria@investigacionenlaescuela.es 


\section{Investigación en la escuela}

Consejo de dirección: Ana Rivero García (Universidad de Sevilla), Nicolás de Alba Fernández (Universidad de Sevilla), Pedro Cañal de León (Universidad de Sevilla), Francisco F. García Pérez (Universidad de Sevilla), Gabriel Travé González (Universidad de Huelva), Francisco F. Pozuelos Estrada (Universidad de Huelva)

Dirección: Ana Rivero García y Nicolás de Alba Fernández

Secretaría de edición: Elisa Navarro Medina

\section{Consejo editorial}

José Félix Angulo Rasco. Universidad de Cádiz Rosa Ma Ávila Ruiz. Universidad de Sevilla Pilar AzcárateGoded. Universidad de Cádiz Juan Bautista Martínez Rodríguez. Universidad de Granada

Nieves Blanco García. Universidad de Málaga Fernando Barragán Medero. Universidad de La Laguna José Carrillo Yáñez. Universidad de Huelva José Contreras Domingo. Universidad de Barcelona. Luis C. Contreras González. Universidad de Huelva Ana $\mathbf{M}^{\mathbf{a}}$ Criado García-Legaz. Universidad de Sevilla Rosario Cubero Pérez. Universidad de Sevilla José $\mathbf{M}^{\mathbf{a}}$ Cuenca López. Universidad de Huelva Jesús Estepa Giménez. Universidad de Huelva Rafael Feito Alonso. Universidad Complutense (Madrid)

Francisco José García Gallardo. Universidad de Huelva

Soledad García Gómez. Universidad de Sevilla J. Eduardo García Díaz. Universidad de Sevilla
Fernando Hernández Hernández. Universidad de Barcelona

Salvador Llinares Ciscar. Universidad de Alicante Alfonso Luque Lozano. Universidad de Sevilla Rosa Martín del Pozo. Universidad Complutense (Madrid)

José Martín Toscano. IES Fernando Herrera (Sevilla) Jaume Martínez Bonafé. Universidad de Valencia F. Javier Merchán Iglesias. Universidad de Sevilla Emilia Moreno Sánchez. Universidad de Huelva. Rosario Ortega Ruiz. Universidad de Córdoba Antonio de Pro Bueno. Universidad de Murcia Fco. de Paula Rodríguez Miranda. Universidad de Huelva

Pedro Sáenz-López Buñuel. Universidad de Huelva Antoni Santisteban Fernández. Universidad Autónoma (Barcelona)

Emilio Solís Ramírez. Catedrático de IES. $\mathbf{M}^{\mathbf{a}}$ Victoria Sánchez García. Universidad de Sevilla. Magdalena Suárez Ortega. Universidad de Sevilla

\section{Consejo asesor}

Manuel Área Moreira. Universidad de La Laguna Jaume Carbonell. Director Cuadernos de Pedagogía. Barcelona César Coll. Universidad de Barcelona

Christopher Day. Universidad de Nothingham. U.K.

Juan Delval. Universidad Nacional de Educación a Distancia

John Elliott. Universidad de East Anglia. Norwich. U.K.

José Gimeno Sacristán. Universidad de Valencia

André Giordan. Universidad de Paris VII y Ginebra

Francisco Imbernón. Universidad de Barcelona Ángel Pérez Gómez. Universidad de Málaga

Rafael Porlán Ariza. Universidad de Sevilla

Francesco Tonucci. Instituto de Pedagogía del C.N.R. Roma

Jurjo Torres Santomé. Universidad de A Coruña 
